\title{
A Programmable Framework for Validating Data Planes
}

\author{
Pietro Bressana \\ Università della Svizzera italiana \\ pietro.bressana@usi.ch
}

\author{
Noa Zilberman \\ University of Cambridge \\ noa.zilberman@cl.cam.ac.uk
}

\author{
Robert Soulé \\ Università della Svizzera italiana \\ robert.soule@usi.ch
}

\begin{abstract}
Due to the emerging trend of programmable network hardware, developers have begun to explore ways to accelerate various applications and services. As a result, there is a pressing need for new tools and techniques for debugging network devices. This paper presents NetDebug, a fully programmable hardware-software framework for validating and real-time debugging of programmable data planes. We describe validation use cases, compare our design to alternative solutions, and present a preliminary evaluation using a prototype implementation.
\end{abstract}

\section{CCS CONCEPTS}

- Networks $\rightarrow$ Programmable networks; In-network processing; • Hardware $\rightarrow$ Networking hardware; Bug detection, localization and diagnosis;

\section{KEYWORDS}

Data plane validation; Programmable networks; Networking hardware

\section{ACM Reference Format:}

Pietro Bressana, Noa Zilberman, and Robert Soulé. 2018. A Programmable Framework for Validating Data Planes. In SIGCOMM Posters and Demos '18: ACM SIGCOMM 2018 Conference Posters and Demos, August 20-25, 2018, Budapest, Hungary. ACM, New York, NY, USA, 3 pages. https://doi.org/10.1145/3234200.3234220

\section{INTRODUCTION}

The way in which we use network devices is changing. The emerging trend of programmable data planes [2] has lead to

\footnotetext{
Permission to make digital or hard copies of all or part of this work for personal or classroom use is granted without fee provided that copies are not made or distributed for profit or commercial advantage and that copies bear this notice and the full citation on the first page. Copyrights for components of this work owned by others than ACM must be honored. Abstracting with credit is permitted. To copy otherwise, or republish, to post on servers or to redistribute to lists, requires prior specific permission and/or a fee. Request permissions from permissions@acm.org.

SIGCOMM Posters and Demos '18, August 20-25, 2018, Budapest, Hungary

(C) 2018 Association for Computing Machinery.

ACM ISBN 978-1-4503-5915-3/18/08 . .\$15.00

https://doi.org/10.1145/3234200.3234220
}

increased interest in in-network computing, a form of hardware acceleration in which applications and services traditionally running on servers are executed on network devices [5, 6]. This begs the question: Given that in-network computing is asking network devices to do so much more work, how can we be sure that they behave correctly?

Validating the correctness of software applications is widely regarded as a difficult task [3]. The challenges become more acute when moving programs to the network hardware, for several reasons. First, the separation of the control plane and data plane makes it hard to reason about the exact configuration in which a program is run. Second, debugging network devices often depends on network traffic. Once a switch stops sending packets, existing tools have no way to diagnose error. Third, network programming languages, such as P4 [4], are designed to be target-independent by abstracting away architecture-specific details. Although this increases code portability, it can lead to problems; code may have undefined behavior on some targets, and compiler may support only a subset of the language specification.

There is clearly a need for new tools and techniques to support network debugging. To address these challenges, we introduce NetDebug, a programmable hardware-software framework, which provides validation and real-time debugging of programmable data planes.

\section{SYSTEM OVERVIEW}

NetDebug provides a fully programmable test infrastructure inside network devices. It allows debugging in real time at full line rate. NetDebug is deployed in parallel to live traffic. Developers write test and validation code using P4. We chose P4 because it was designed specifically for network programming and has been widely adopted on a number of hardware platforms [7-9]. However, NetDebug is independent from the language of the application. It can validate data planes designed using a number of different workflows and languages, including high level synthesis, $\mathrm{P} 4, \mathrm{C} / \mathrm{C} \#$ and hardware description languages.

The NetDebug architecture, shown in Figure 1, is composed of two hardware modules implemented inside the target network platform: a test packet generator and an output packet checker. Both modules are managed by a software tool, running on a host computer, which uses a dedicated 


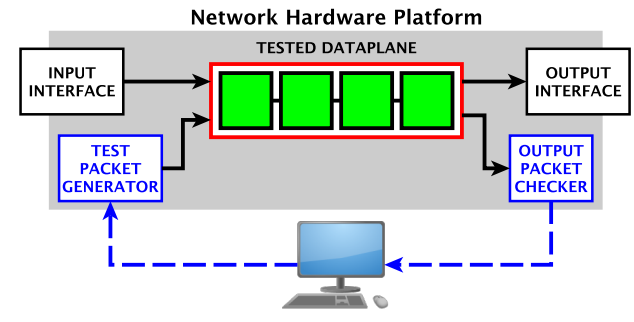

Figure 1: The proposed architecture

interface to configure the generation of test packets and to collect test results. The hardware infrastructure, which is fully programmable through $\mathrm{P} 4$ language, is internally connected to the data plane under test. This design allows NetDebug to validate the data plane while avoiding the surrounding hardware, including the network interfaces. Users can generate custom test packets and inject them directly into the data plane under test, while running verification on output packets at line rate, at real time. This is specifically beneficial for programmable data planes, where the same architecture is used for many different programs. If a bug prevents packets from being correctly forwarded to the output interfaces of the device, users can find where the fault occurred, even inside the data plane.

\section{USE-CASES}

NetDebug can be applied to a wide range of use cases, including but not limited to:

- Functional testing: finding functional bugs in the data plane and in the control plane

- Performance testing: performance metrics, such as throughput, packet rate and latency

- Compiler check: finding limitations in the compiler

- Architecture check: finding limitations in the architecture

- Resources quantification: evaluating the consumption of hardware resources

- Status monitoring: providing periodic internal status information

- Comparison: comparing alternative specifications of the same program

Figure 2 summarizes the capabilities of NetDebug compared to related work. Software formal verification tools [3] can check functional correctness. They can not be used to test a hardware implementation, since verification is performed only on the software specification of the programs. External network testers [1] are able to run only partial tests related to functional, performance, compiler and architecture use-cases, since they lack a "internal view" of the device under test, as they are limited by the external interfaces of a device,

\begin{tabular}{|c||c||c||c|}
\hline Use-case & NetDebug & $\begin{array}{c}\text { Software } \\
\text { formal verifiers }\end{array}$ & $\begin{array}{c}\text { External } \\
\text { network testers }\end{array}$ \\
\hline \hline $\begin{array}{c}\text { Functional } \\
\text { testing }\end{array}$ & $\checkmark$ & $\checkmark$ & partial \\
\hline $\begin{array}{c}\text { Performance } \\
\text { testing }\end{array}$ & $\checkmark$ & & partial \\
\hline $\begin{array}{c}\text { Compiler } \\
\text { check }\end{array}$ & $\checkmark$ & & partial \\
\hline $\begin{array}{c}\text { Architecture } \\
\text { check }\end{array}$ & $\checkmark$ & & \\
\hline $\begin{array}{c}\text { Resources } \\
\text { quantification }\end{array}$ & $\checkmark$ & & \\
\hline $\begin{array}{c}\text { Status } \\
\text { monitoring }\end{array}$ & $\checkmark$ & partial & partial \\
\hline Comparison & $\checkmark$ & & \\
\hline
\end{tabular}

Figure 2: Use-cases

and have no visibility into internal data plane failures. For the same reason, they are unable to test either the resources or status monitoring use-cases. NetDebug can perform full comparisons, since it is able to run tests related to all the discussed use-cases. Software formal verification tools and external network testers can make comparisons based on the use-cases that they are able to test.

\section{EVALUATION}

We have implemented a prototype of NetDebug on NetFPGA SUME [11] using Xilinx SDNet [10], which translates P4 specifications into a hardware module. We have used NetDebug to debug several data plane programs.

Preliminary experiments have already provided some useful insights. For example, using NetDebug, we discovered that the reject parser state, an essential feature of $\mathrm{P} 4$ language, is not implemented by SDNet. This meant that any packet coming into the data plane was sent out to the next hop, even if it was supposed to be dropped. Our framework immediately detected this severe bug, that would not be noticed by applying software formal verification to the data plane program [3].

\section{CONCLUSIONS}

We have presented NetDebug, a programmable framework for validating data planes. NetDebug leverages both the P4 language and hardware design to provide flexibility and visibility into programmable network devices. We have built a prototype of NetDebug, and used it to detect a bug not visible through software formal verification tools. As innetwork computing becomes increasingly popular, NetDebug addresses an urgent need for improved tools and techniques for data plane debugging and verification.

Acknowledgments This work is partially supported by SNSF award 407540_167173, Doc.Mobility fellowship P1TIP2_178657, the Leverhulme Trust (ECF-2016-289), and the Isaac Newton Trust. 


\section{A Programmable Framework}

for Validating Data Planes

\section{REFERENCES}

[1] G. Antichi, M. Shahbaz, Y. Geng, N. Zilberman, A. Covington, M. Bruyere, N. Mckeown, N. Feamster, B. Felderman, M. Blott, A. W. Moore, and P. Owezarski. September 2014. OSNT: Open Source Network Tester. IEEE Network (September 2014).

[2] P. Bosshart, G. Gibb, H. Kim, G. Varghese, N. McKeown, M. Izzard, F. Mujica, and M. Horowitz. August 2013. Forwarding Metamorphosis: Fast Programmable Match-action Processing in Hardware for SDN SIGCOMM Computer Communication Review (CCR) (August 2013).

[3] C. Cascaval, N. Foster, W. Hallahan, J. Lee, J. Liu, N. McKeown, C. Schesinger, M. Sharif, R. Soulé, and H. Wang. August 2018. p4v: Practical Verification for Programmable Data Planes. ACM SIGCOMM Conference on Applications, Technologies, Architectures, and Protocols for Computer Communications (SIGCOMM) (August 2018).

[4] The P4 Language Consortium. May 2017. " $\mathrm{P} 416$ Language specification (v1.0.0)". https://p4.org/p4-spec/docs/P4-16-v1.0.0-spec.html

[5] X. Jin, X. Li, H. Zhang, N. Foster, J. Lee, R. Soulé, C. Kim, and I. Stoica. April 2018. NetChain: Scale-Free Sub-RTT Coordination. In 15th USENIX Symposium on Networked Systems Design and Implementation (NSDI).

[6] X. Jin, X. Li, H. Zhang, R. Soulé, J. Lee, N. Foster, C. Kim, and I. Stoica. October 2017. NetCache: Balancing Key-Value Stores with Fast InNetwork Caching. In Proceedings of the 26th Symposium on Operating Systems Principles (SOSP).

[7] Netronome. June 2018. Netronome SmartNICs. https://www. netronome.com/

[8] Barefoot Networks. June 2018. Barefoot Tofino. https:// barefootnetworks.com/products/brief-tofino/

[9] Xilinx. June 2018. What is an FPGA? https://www.xilinx.com/ products/silicon-devices/fpga/what-is-an-fpga.html

[10] Xilinx. June 2018. Xilinx SDNet. https://www.xilinx.com/products/ design-tools/software-zone/sdnet.html

[11] N. Zilberman, Y. Audzevich, G. A. Covington, and A. W. Moore. September 2014. NetFPGA SUME: Toward 100 Gbps as Research Commodity. IEEE Micro (September 2014). 